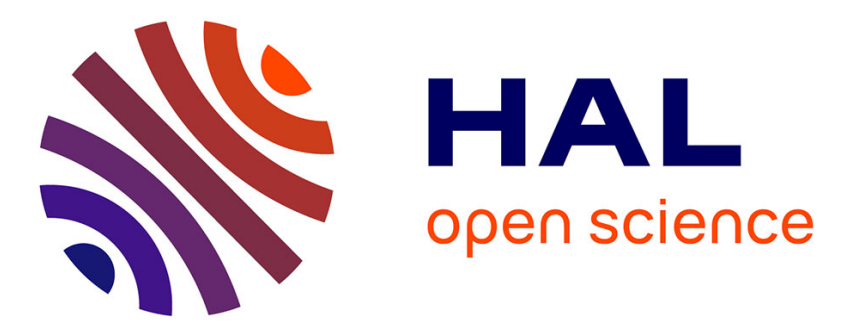

\title{
Thermo-rheological-kinetical Study of Compression Molding of Fibre-reinforced Composites
}

\author{
L F Salazar Betancourt, P Laure, L F Silva, M F Sager
}

\section{To cite this version:}

L F Salazar Betancourt, P Laure, L F Silva, M F Sager. Thermo-rheological-kinetical Study of Compression Molding of Fibre-reinforced Composites. ESAFORM 2016, Apr 2016, Nantes, France. pp.170013, 10.1063/1.4963569 . hal-01385483

\section{HAL Id: hal-01385483 \\ https://hal.science/hal-01385483}

Submitted on 21 Oct 2016

HAL is a multi-disciplinary open access archive for the deposit and dissemination of scientific research documents, whether they are published or not. The documents may come from teaching and research institutions in France or abroad, or from public or private research centers.
L'archive ouverte pluridisciplinaire HAL, est destinée au dépôt et à la diffusion de documents scientifiques de niveau recherche, publiés ou non, émanant des établissements d'enseignement et de recherche français ou étrangers, des laboratoires publics ou privés. 


\title{
Thermo-rheological-kinetical Study Of Compression Molding Of Fibre-reinforced Composites
}

\author{
L.F. Salazar Betancourt ${ }^{1, \mathrm{a}), \mathrm{b})}$, P. Laure ${ }^{1, \mathrm{c})}$, L. Silva ${ }^{2, \mathrm{~d})}$ and M. Sager ${ }^{3, \mathrm{e})}$ \\ ${ }^{1}$ MINES ParisTech, PSL - Research University, CEMEF - Centre for material forming, CNRS UMR 7635, CS 10207 \\ rue Claude Daunesse 06904 Sophia Antipolis Cedex, France. \\ ${ }^{2}$ ICI - ECN Ecole Centrale Nantes 1 Rue de la Noe, 44300 Nantes, France \\ ${ }^{3}$ Plastic Omnium AR Services, Place de l'ain avanue du Bois des Vergnes, 01150 Sainte Julie, France \\ a)Corresponding author: luis-fernando.salazar_betancourt@ mines-paristech.fr \\ ${ }^{b)}$ URL: http://www.cemef.mines-paristech.fr \\ ${ }^{c}$ patrice.laure@mines-paristech.fr \\ d)luisa.rocha-da-silva@ec-nantes.fr \\ e) mustafa.sager@plasticomnium.com
}

\begin{abstract}
To improve the modeling of fiber reinforced composites, we present in this work numerical methods able to compute both fiber-reinforced composites deformation in squeeze flow and thermal-kinetic evolution. The rheology is given by an homogeneous orthotropic model for fiber composites which describes the anisotropy of the in-plane fiber. The thermics is then extended accounting for the reaction here formulated by the Bailleul's model. Both physics are related since the kinetic evolution as well as the temperature profile modify the rheology of the composites, giving raise to the thermo-rheological-kinetical coupling by means of the viscosity temperature dependence. A study case is presented, where the mold temperature is set to $150^{\circ} \mathrm{C}$ with a composite sample at $40^{\circ} \mathrm{C}$. Thermal transfer begins as well as sample compression at constant speed. We present the evolution of the reaction, temperature and viscosity at the core and the surface. Reaction in the core of the material is much quicker than in the surface. Which means that a mapping of viscosity values is presented during the reaction modifying the mechanical response.
\end{abstract}

\section{INTRODUCTION}

Fiber-reinfroced Composites materials represent an interesting approach for lighting automotive structural pieces. The mechanical modeling of such materials has started with the work of Barone et al [1] improved with the study performed by Kotsikos et al [2]. Nevertheless, it was until the work of Le Corre [3] when its anisotropic modeling began. Later, Dumont et al in [4] proposed a model that accounts for the anisotropic behavior exhibited by reinforced materials. By means of structural tensors a full formulation is presented allowing its direct numerical implementation as shown in [5]. However, current models taking into account the thermo-kinetic nature of such composites remains a complementary key towards the thermo-mechanical modeling of reinforced parts. First studies were accomplished by Lee in [6] and by Maazouza [7] using a model based on the radical polymerisation mechanisms. Currently adapted models to upcoming reinforced materials are needed. Models describing the conversion degree evolution of the composites as well as its direct link to mechanical response.

In this work a thermo-rheological-kinetical computation of the heating-compression of a reinforced sample is presented. In this study we observe not only the squeeze-flow mechanics characteristic of this process, but also the thermal evolution of the sample, including resin reaction. Both, thermal and kinetic evolution are coupled to the viscosity of the material. Thus, affecting the squeeze-flow nature.

\section{MODELING FIBRE-REINFORCED COMPOSITES IN IMMERSED DOMAINS}

Compression molding processes involve more complex physical phenomena than solving a fluid mechanics problem. In fact, modeling these processes come along with studying the coupling between the mechanical problem, the thermal 

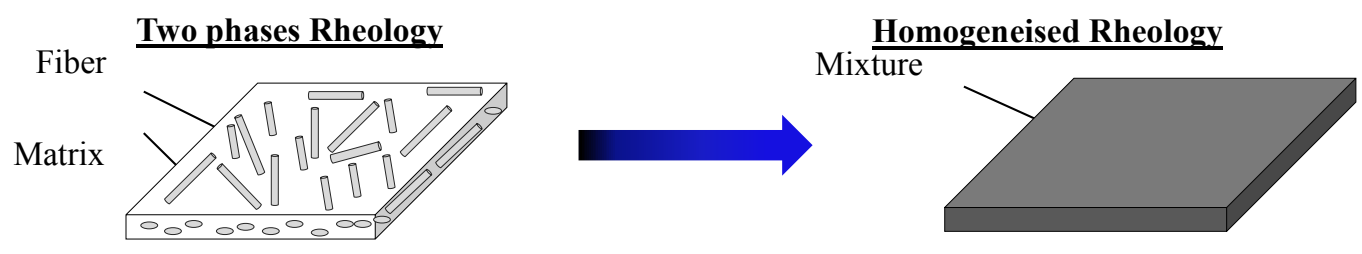

FIGURE 1. Fibre reinforced composites mainly described by two phases are described here as a homogeneous mixture characterized by its fiber fraction $f$. All the parameters and variables here described represents the mixture of both phases.

one and for thermosets, the polymerization or degree of curing reaction.

\section{Governing Equations}

Let us consider the homogeneous flow of a fiber reinforced composite fluid (Fig.1) described by the Navier-Stokes equations, with an orthotropic non-Newtonian compressible constitutive law associated to appropriates boundary conditions. We extend the modeling here by adding the thermal evolution of the sample by describing not only its thermal state but also its thermo-kinetics due to resin composition by means of the term $q_{\text {react }}$. We also included in this modeling the evolution of the conversion degree $\alpha$, which accounts for the measurement of the reaction level. One writes the governing equations of the fully modeling as follows:

$$
\begin{aligned}
\rho\left(\frac{\partial \vec{v}}{\partial t}+\vec{v} \cdot \nabla \vec{v}\right)-\nabla \cdot \underline{\sigma} & =\rho \vec{g} \\
\nabla \cdot \vec{v} & =0 \\
\rho C p\left(\frac{\partial T}{\partial t}+\vec{v} \cdot \nabla T\right)-\nabla \cdot(\kappa \nabla T)-\underline{\sigma}: \nabla \vec{v} & =\dot{q}_{\text {react }} \\
\frac{\partial \alpha}{\partial t}+\vec{v} \cdot \nabla \alpha & =F(\alpha, T)
\end{aligned}
$$

with $\vec{v}$ the velocity of the mixture, $\rho$ the density of the mixture, $\sigma$ the stress Cauchy stress tensor and $\vec{g}$ the force of gravity. The thermal parameters $C_{p}$ states for the heat capacity of the mixture, $\kappa$ the conductivity, $\dot{q}_{\text {reac }}$ the heat released by the reaction and $F(\alpha, T)$ the evolution function of the conversion degree.

\section{Immersed Approach}

In this work, the level set method for tracking the composite interface $\Gamma$ is used [8]. Particularly, the linearized hyperbolic tangent $\psi$ defined in (eq.5). Here, $d(x, \Gamma)$ stands for the distance of a point to the interface, $e$ is the thickness used to mix the physical properties along the domain and $E$ a thickness where the level set function is truncated. Function $\psi$ is convected to described the interface movement adding a reinitialization velocity $\vec{v}_{r}$ conserving the metric properties of the function (eq.6).

$$
\psi=\left\{\begin{array}{lll}
e+E \tanh \left(\frac{\mathrm{d}(\mathrm{x}, \Gamma)-e}{E}\right) & \text { if } \quad \psi>e \\
\pm \mathrm{d}(\mathrm{x}, \Gamma) & \text { if } \quad-e<\psi<e \\
-e+E \tanh \left(\frac{-\mathrm{d}(\mathrm{x}, \Gamma)+e}{E}\right) & \text { if } \quad \psi<-e
\end{array} \quad \frac{\partial \psi}{\partial t}+\left(\vec{v}+\vec{v}_{r}\right) \cdot \nabla \psi=f(\psi)\right.
$$

When solving (eq.6) volume conservation is not guarantee. For correcting this lost, a predictor-correction scheme is used. Conservation implies $\int_{\Omega} H(\phi) d \Omega-V_{0}=0$, where $V_{0}$ is the initial volume and $H=1 / 2+\psi / 2 E$, the smoothed Heaviside function associated to the level set. First we computed the volume lost each iteration by $f^{n+1}=\int_{\Omega} H(\psi) d \Omega-$ 
$V_{0}$. Then, the conservative level set $\phi$ is obtained by (eq.7). Where $\delta(\alpha)$ is a Dirac function associated to level set [9].

$$
\phi^{n+1}=\psi-\frac{f^{n+1}}{\int_{\Omega} \delta(\alpha) d \Omega} .
$$

In this strategy, the correction is not space dependent. For more enriched methods the reader is referred to [10]. However, we take into account the presence of the mold and punch (tools) in order to not apply the correction causing penetration of the level set into the tools.

\section{Thermo-rheological-kinetical coupling}

In this section, we present the expression of the stress tensor $\sigma$ used in (eq.1), that takes into account the anisotropy nature of the fiber network. Additionally, the expression of the heat rate $q_{\text {react }}$ used in (eq.3) is given. These energy released by the reaction represents the transformation of the chemical energy of the resin into heat. Both natures are related through the viscosity expression. During the compression forming, the pseudo-plastic viscosity decreases with the strain rate and the high temperature. However, the evolution of the kinetic trigger the polymer chains and entangled them causing the solidification of the piece, translating into a viscosity increment.

\section{Rheology Model}

The rheological model used in this work is an extension of the model found in [4]. The stress tensor is defined by (eq.8) where the anisotropy of the composite is described by the extra contributions associated to the deformation strain rate $\underline{D}$ described in (eq.9), by the tensor $\underline{M}$ and the parameters $\alpha_{0}=1.03, \alpha_{1}=1.446, \alpha_{2}=-1.97$. $p$ states for the incompressible pressure.

$$
\begin{aligned}
\underline{\sigma} & =\alpha_{0} \eta_{e q}\left[\underline{D}+\alpha_{1}(\underline{M}: \underline{D}) \underline{M}+\frac{\alpha_{2}}{2}(\underline{M} \cdot \underline{D}+\underline{D} \cdot \underline{M})\right]-p \underline{I} \\
\underline{D} & =\frac{1}{2}\left(\nabla \vec{v}+\nabla^{t} \vec{v}\right) \\
D_{e q}^{2} & =\alpha_{0}\left(\underline{D}: \underline{D}+\alpha_{1}(\underline{M}: \underline{D})^{2}+\alpha_{2}(\underline{D} \cdot \underline{M}: \underline{D})\right)
\end{aligned}
$$

The model assumes plug flow, causing fibers to spread in an in-plane way. Averaging the fibers' directions modify homogeneously the macroscopic stress, leading to plane isotropy. Here, $\eta_{e q}$ states for the consistency of the paste under a concentration $f$ of fibers, and states for its power-law exponent $m$ and the equivalent strain rate $D_{e q}$, here defined as shown in (eq.10). The orthogonal direction $n$ represents the normal to the fibers' in-plane motion (here, the vertical direction) and its effect is represented in the model by means of the tensor $\underline{M}=n \otimes n$. Parameters $\alpha$, account for fiber concentration $f$ and expressions for these values are found in the original paper [4]. In addition, a numerical comparison of the response of the stress tensor for simple compression, plane strain and shearing cases without taking into account kinetics was performed in [5].

\section{Thermo-kinetic Model}

The kinetic model is giving through the heat released $\dot{q}_{\text {react }}$ computed by means of the Bailleul's Model. Where the heat released is written by (eq.11):

$$
\dot{q}_{\text {reac }}(T, \alpha)=\rho(T, \alpha) \frac{d \alpha}{d t} \Delta H_{\text {tot }} .
$$

The computation of the evolution of the reaction rate $F(\alpha, T)$ (eq.12) is usually described by the product of a temperature function $K(T)$, a function depending on the conversion degree $G(\alpha)$ and the induction period function $h(T)$ all described in (eq.13). The activation function $W(h(t))$ stands to zero if the ignition period has not been reached yet and for 1 once all the inhibitors of the resin has been consumed. These expressions read: 


$$
\begin{aligned}
F(\alpha, T) & =W(h(t)) K(T) G(\alpha), \\
K(T)=k_{r e f} \exp \left(-A\left(\frac{T_{r e f}}{T}-1\right)\right) ; \quad G(\alpha) & =\sum a_{i} \alpha^{i} ; \quad h(T, t)=t_{r e f}-\int_{0}^{t} \exp \left(-B\left(\frac{T_{r e f}}{T}-1\right)\right) d t .
\end{aligned}
$$

In general, all thermal parameter depend not only on temperature $T$ but also on the conversion degree $\alpha$. From a numerical perspective, we focus on the modeling of the thermo-rhelogical-kinetic coupling of fiber reinforced materials. Expressions for the parameters $k_{r e f}, A, T_{r e f}, a_{i}, t_{r e f}, B$ depend on the resin composition of the composites. Expressions for the evolution of the thermal parameter $C p, \rho, \kappa$ are not shown in here, due to confidentiality.

\section{Coupling with viscosity}

The thermo-kinetic-mechanical coupling is done by factors $h\left(D_{e q}\right), f(T)$ and $g(\alpha)$. The viscosity $\eta_{e q}$ is then calculated via $\eta_{e q}=\eta_{0} h\left(D_{e q}\right) f(T) g(\alpha)$. Here $f(T)$ yields for the coupling with the thermal evolution, $g(\alpha)$ the kinetic coupling and $h\left(D_{e q}\right)$ the pseudo plastic coupling. Expression for those contributions are presented in $($ eq. 14, 15, 16) :

$$
\begin{aligned}
h\left(D_{e q}\right) & =\left(1+98 f+980 f^{2}\right)\left(2 \frac{1+H}{1+2 H}\right)^{-\frac{m+1}{2}} D_{e q}{ }^{m-1} \\
f(T) & =e^{b\left(\frac{1}{T}-\frac{1}{T o}\right)} \\
g(\alpha) & =\left(1-\frac{\alpha}{\alpha_{g e l}}\right)^{s}+g_{\text {min }}
\end{aligned}
$$

The pseudo plastic coupling depends on the fiber fraction $f=14.7 \%$ and rheological parameters $H=0.4735, L=$ 46.87 and the sensibility on the strain rate $m=0.44$. The thermal coupling follows an Arrhenius law type depending on activation energy $b=4500 \mathrm{~K}$ and reference temperature $T o=296 \mathrm{~K}$. The kinetical coupling is giving by the evolution of the conversion degree translating into the solidification of the piece. Here, the coupling depends on $\alpha_{g e l}=0.05$ the reference conversion degree and $s=2.0$ a hardening factor depending on the nature of the polymer composites. Here, $g_{\min }=0.1$ states for a minimum kinetic factor when the conversion degree equals $\alpha_{g e l}$.

\section{COMPRESSION MOLDING OF FIBRE REINFORCED COMPOSITES}

As example of fully coupled resolution, we present a test case where a sample is compressed at constant velocity (Fig. 2). The Punch and the Mold have a temperature of $150{ }^{\circ} \mathrm{C}$, whereas the sample has an initial temperature of $40{ }^{\circ} \mathrm{C}$. The sample is compressed at a constant speed of $v=0.004 \mathrm{mms}^{-1}$ while being heat up.

\begin{tabular}{|l|l|}
\multicolumn{1}{l|}{ Punch } & \multicolumn{1}{c|}{$T_{\text {imposed }}=150^{\circ} \mathrm{C}$} \\
\hline & contact layer \\
\hline \multicolumn{1}{|c|}{ Air $\mathrm{T}=25^{\circ} \mathrm{C}$} & $\mathrm{T}=40^{\circ} \mathrm{C}, 15 \%$ fibers \\
\hline & contact layer \\
\hline Mold & $T_{\text {imposed }}=150^{\circ} \mathrm{C}$ \\
\hline
\end{tabular}

FIGURE 2. Compression Molding of composite sample $40 \mathrm{~mm} \times 6 \mathrm{~mm}$. Mold and Punch tool at $150 \mathrm{C}$. Sample with initial temperature of $40^{\circ} \mathrm{C}$. Sample is compressed at constant speed $v$ during reaction.

The simulation depicts the evolution of the viscosity during the polymerization of the composite. As compression occurs, the hot plates heat up the sample until reaction is triggered. The temperature, shearing and the polymerization interacts modifying the viscosity of the sample. A mesh adaptation technique is implemented, bounded for a minimum mesh size of $h_{\min }=0.08 \mathrm{~mm}$. Mesh is adapted respect to velocity, temperature and level set fields. An adaptive time step is bounded to avoid a change in temperature per node higher than $5^{\circ} \mathrm{C}$. Also, the time step fulfill the CFL condition for the resolution of (eq. 6). 


\section{Evolution of the Conversion Degree}

In order to analyze the reaction mechanism, we present here the evolution of the conversion degree $\alpha$. As evidenced in Fig. 3 the reaction begins at time about 200s for the surfaces and at time equals 380s in the core. The evolution of the $\alpha$ value is well described by our discrete temporal scheme. The reaction in the core of the material occurs faster than in the surface, this due to the higher heat transfer in the center coming from the neighboring. Whereas the reaction on the surface described an extended hyperbolic tangent function, the reaction in the core describe basically a more narrow function. The reaction in the core last really few seconds compare to the reaction in the surface which is smooth. This mechanism is captured by our numerical scheme due to our adaptive time step.

Heat transfer and reaction

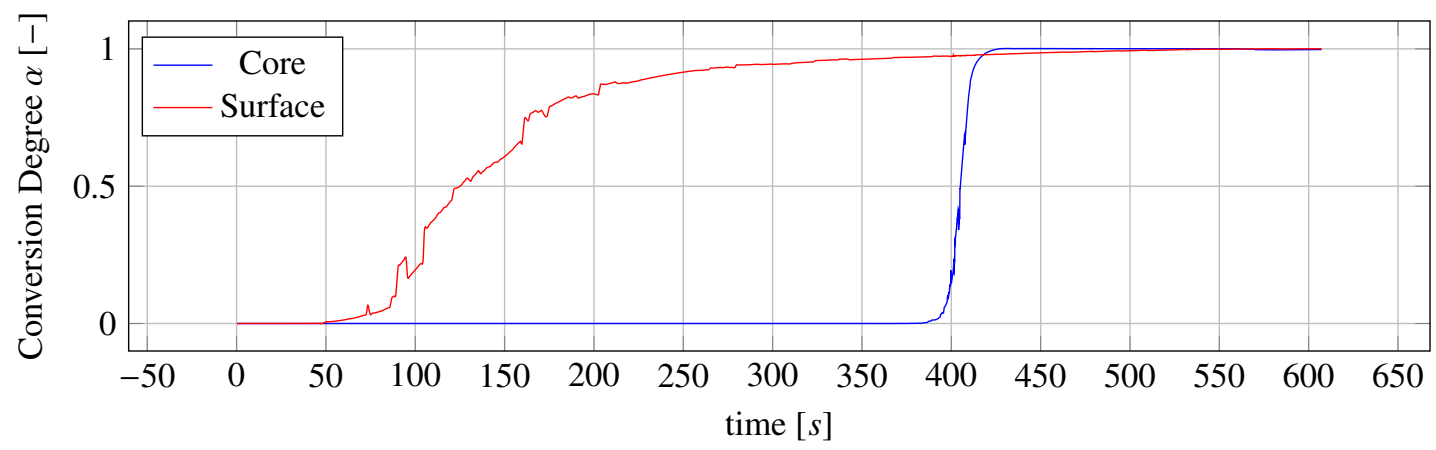

FIGURE 3. Evolution of the conversion degree

\section{Evolution of the temperature}

In Fig. 4, the temperature evolution on the surface and on the center during the heating is depicted. We observe how in the core, temperature remains under the surface temperature until reaction takes place. The heat released is then concentrated in the core causing the temperature of the core to be the highest of the system. increasing even $50{ }^{\circ} \mathrm{C}$ more than the temperature of the hot plates. Once reaction is consumed, the thermal balance is achieved towards the temperature imposed by the plates.

Heat transfer and reaction

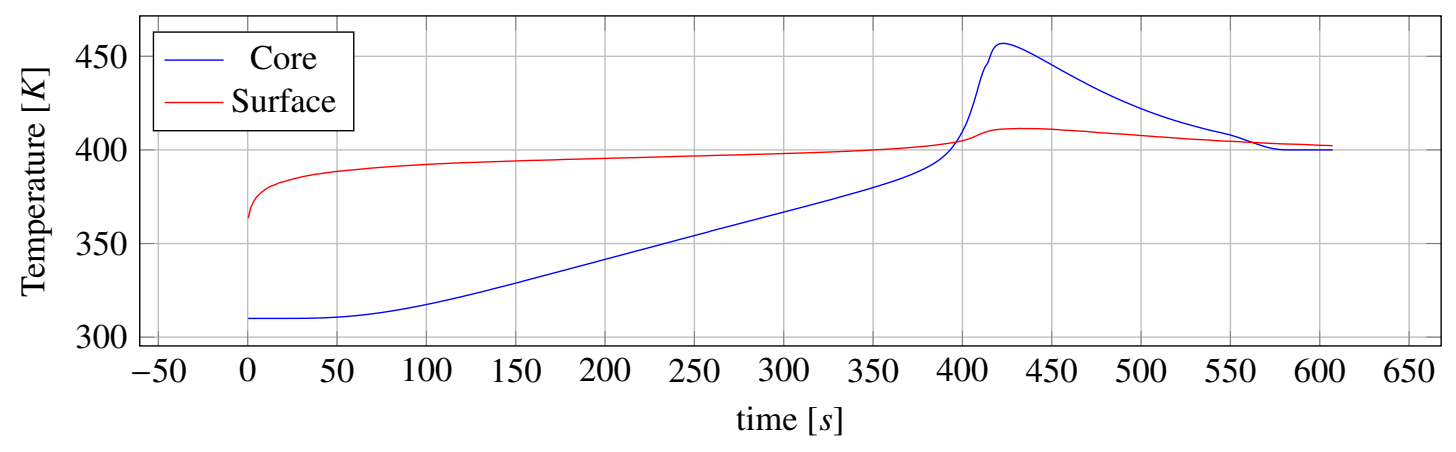

FIGURE 4. Temperature profile during temperature surface imposition. Temperature in the core shows the reaction kinetics.

\section{Evolution of the viscosity}

The thermal and kinetic evolution of the sample make the viscosity evolves. The increase in temperature tends to decrease the viscosity whereas the higher the conversion degree, the viscosity increases since solidification takes 
place. In Fig5 we compare the evolution of viscosity in the surface and the core. Basically this evolution show the combination of the shearing, thermal, and polymerization process. During about 250 s the surface viscosity decreases for the thermal heating and just then raises up. The core of the sample raises up the viscosity much later than the surface. We conclude in this test case that conversion degree governs the viscosity of the sample.

Evolution of the viscosity through reaction and thermal evolution

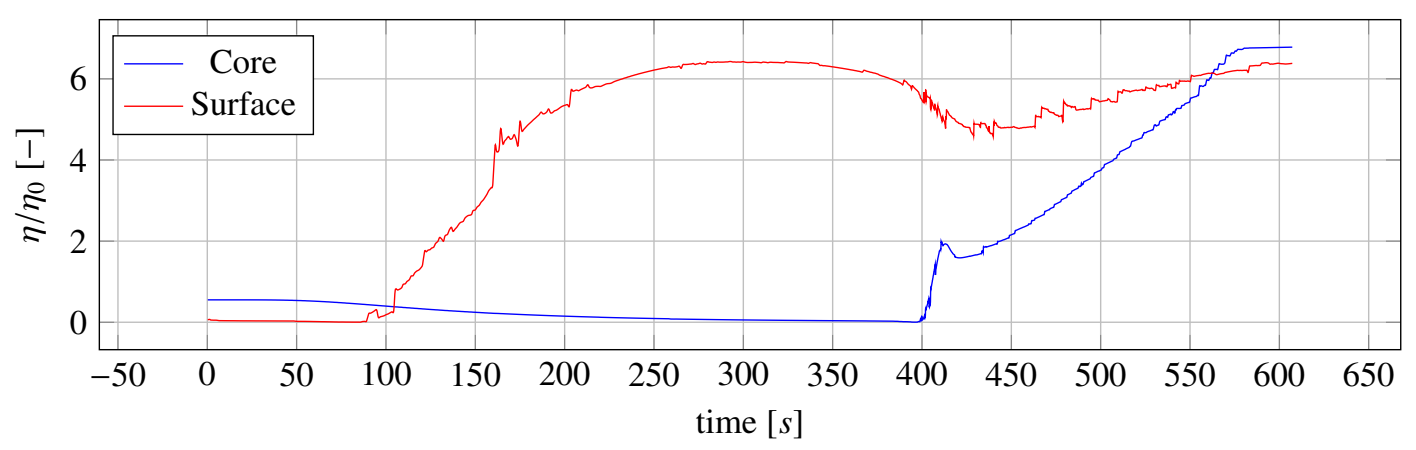

FIGURE 5. Viscosity evolution during compression and heating of the sample. viscosity in the surface and in the core shows the interaction between the thermal softening and the kinetic hardening.

\section{Impact of thermo-kinetics on mechanical response}

In this section we present the modification of the mechanical response due to the thermo-kinetic coupling. In Fig.6 the temperature profile along the sample shows the mapping of values during the reaction. This picture at $t=250 \mathrm{~s}$ clearly shows the temperature profile during the compression. We see how the heat released by the reaction increases the temperature of the sample to higher values than the imposed temperature.

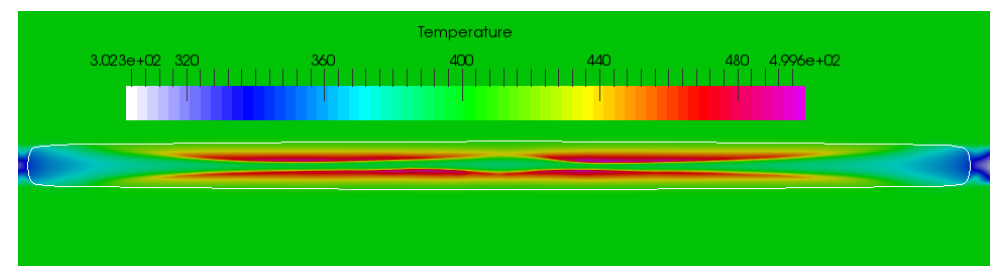

FIGURE 6. Compression molding of reinforced composites. Temperature field along the sample in Kelvins at $t=250 \mathrm{~s}$. We observe the hot zones where the reaction just took place.

For the time of the picture in Fig.7, the solidified shell belong to reaction values of 1 . Whereas, 0 values states for the non-reacted material.

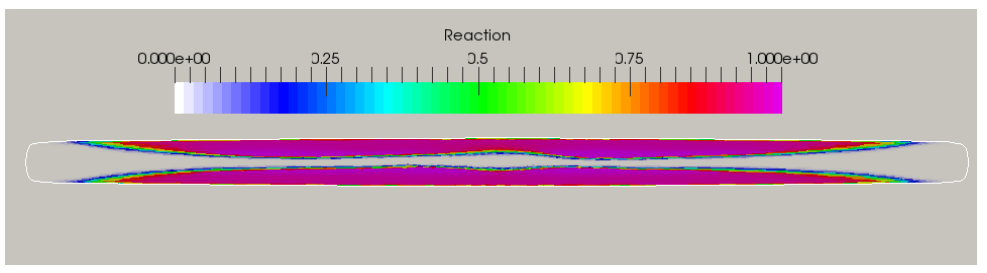

FIGURE 7. Conversion degree $\alpha$. We observe the front of the reaction at $t=250 \mathrm{~s}$. The reaction begins in the surfaces and moves towards the center and towards the external boundaries.

This shape gives us a indicator of how reaction grow in the sample. Begin in the surface, moves towards the center and towards the exterior. 
In Fig. 8 the mapping of viscosity is depicted. Lower viscosity values are found outside of the solidification shell and close to the side extreme, where shearing is higher. The coupled of the thermal factor and the kinetic polymerization modify the viscosity.

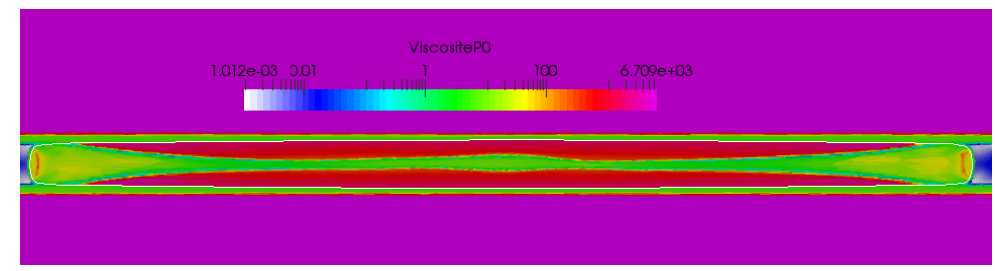

FIGURE 8. Viscosity field along the sample in Pascal. We observed zones with higher viscosity due to solidification at $t=250 \mathrm{~s}$.

Such modification of the viscosity induces different flow than the normal found in squeeze flow fluids. The viscosity mapping affects the velocity and stress development since they are now solidified zones and liquid zones. The velocity gradient are found to be naturally higher in the fluid zone. As example, the velocity in a intermediate point of the reaction is presented in Fig.9. We observe how the velocity profile present strong gradient since part of the piece has solidified. Basically, the solidified shell works as punch and mold squeezing out the fluid towards the exterior.

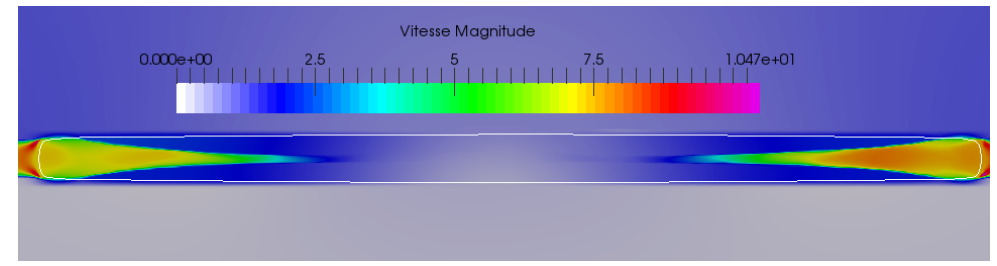

FIGURE 9. Velocity magnitude during reaction in $\mathrm{mm} \mathrm{s}^{-1}$ at $t=250 \mathrm{~s}$. Solidification modifies the viscosity of the sample close to the surfaces. The common squeeze flow is not longer seem. during reaction, the material with lower viscosity tends to be squeezed.

At that point, the stress concentration on the solidified shell is shown in Fig.10. The mapping of green values. The red values are assigned to the liquid phase in the core that get entrapped. The lowest values are found towards the exterior walls, where the shearing is higher and reaction has not yet begun.

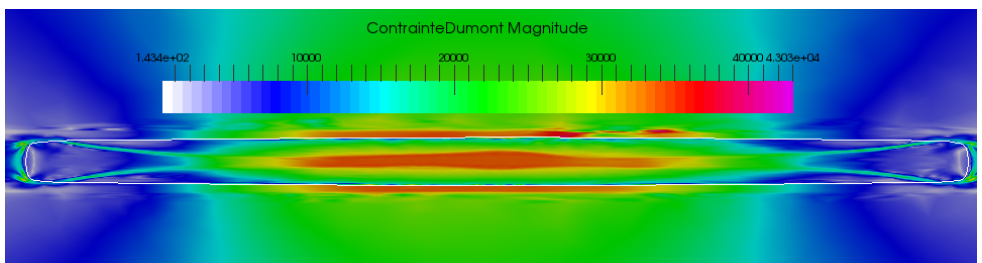

FIGURE 10. Stress magnitude at $t=250 \mathrm{~s}$ in Pascal under reaction during compression.

Normally, during compression molding, we would like to have first the filling of a part before the reaction takes place. However, we have seen in here that reaction in the surface takes place during almost the whole process. Of course, mold temperature plays a roll on the beginning of the reaction, but in this simple example, we can see how the reaction mechanism is depth depend, has different reacting times, has a different rate of conversion and modify the pattern of the flow. Details that should be taking into account in the conception phase of industrial piece designing. A more concluding results can be the evolution of the compression force. In Fig.11 is shown the increment of the force once reaction occurs in the center. At time $t=400 \mathrm{~s}$ the force has a 'jump'. By looking at the time, it can say that the macroscopic force is indeed modify when reaction in the core takes place. Basically it describes the length of the reaction in the core since it present the same shape as the conversion degree in the core described in Fig.3. 
Evolution of the compression force through reaction and thermal evolution

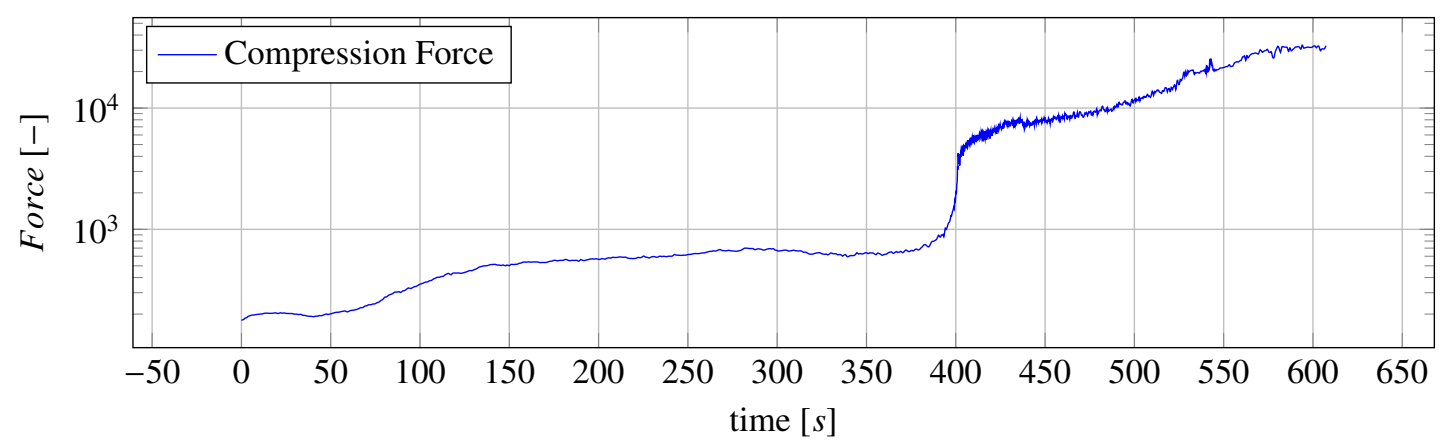

FIGURE 11. Compression Force evolution. When compressing the sample the squeeze force increased by the increasing of the surface in contact. The force slope is affected as well for the hardening due to polymerization.

\section{CONCLUSIONS}

This work has presented a coupled thermo-kinetic-mechanical model of fiber-reinforced composites during compression molding. In here, the rheology of the material is described by an one-equivalent-phase orthotropic model that accounts for the anisotropy exhibits for the fiber-network. For the thermal resolution a thermo-kinetic model is used to describe the polymerization mechanism. A thermo-rheo-mechanical coupling is then formulated by means of factors modifying the viscosity of the composites. A compression molding study case is studied showing the evolution of the viscosity, temperature, velocity and stress during reaction. We evidenced that reaction in the core of the material is much quicker than in the surface. That means that a mapping of viscosity values is presented during the reaction modifying the usual the squeeze flow. For our case, the highest temperature of the system was found in the core where reaction takes place as last but quicker than elsewhere. The reaction mechanism moves from the walls towards the center and extended then to the exterior. Thus, having different reacting times causing space-depend solidification zones. We can conclude as well that the length of the reaction in the core can be estimated by the macroscopic force evolution.

\section{ACKNOWLEDGMENTS}

The authors would like to acknowledge to Romain Cardinaud for providing the kinetic-model used in this work and its advises during the numerical implementation.

\section{REFERENCES}

[1] M. Barone and D. Caulk, Journal of applied mechanics 53, 361-371 (1986).

[2] G. Kotsikos and A. Gibson, Composites Part A: Applied Science and Manufacturing 29, 1569-1577 (1998).

[3] S. Le Corre, L. Orgéas, D. Favier, A. Tourabi, A. Maazouz, and C. Venet, Composites science and technology 62, 571-577 (2002).

[4] P. Dumont, L. Orgéas, S. Le Corre, and D. Favier, International Journal of Plasticity 19, 625-646 (2003).

[5] L. F. Salazar Betancourt, P. Laure, L. Silva, and M. Sager, "Numerical implementation of a rheology model for fiber-reinforced composite and viscous layer approach for friction study," in Key Engineering Materials, Vol. 651 (Trans Tech Publ, 2015), pp. 848-854.

[6] J. Lee Ly, Polymer Engineering \& Science 21-8, 483-492 (1981).

[7] A. M. G. S. V Massardier-Nageottea, F Caraa, Composites Science and Technology 64 issue 12, 1855-1862 (2004).

[8] T. Coupez, AIP Conference Proceedings 908, 61-66 (2007).

[9] S. Zahedi and A.-K. Tornberg, Journal of Computational Physics 229, 2199-2219 (2010).

[10] C. E. Kees, I. Akkerman, M. W. Farthing, and Y. Bazilevs, Journal of Computational Physics 230, 45364558 (2011). 